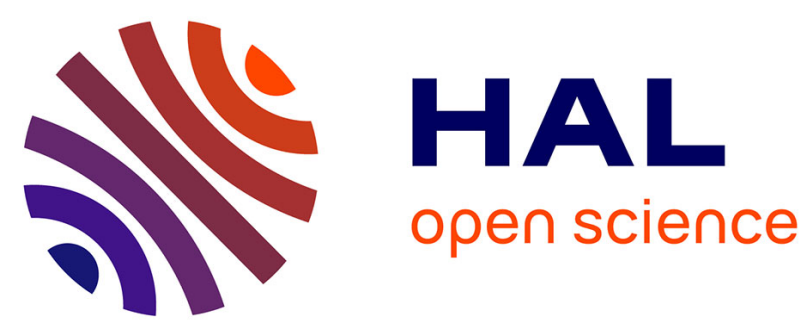

\title{
Etude par EXAFS et fluorescence X de photocatalyseurs zéolithiques renfermant des nanoparticules de $\mathrm{ZnO}$
}

\author{
L. Khouchaf, M. Tuilier, J. Dürr, M. Wark, H. Kessler
}

\section{To cite this version:}

L. Khouchaf, M. Tuilier, J. Dürr, M. Wark, H. Kessler. Etude par EXAFS et fluorescence X de photocatalyseurs zéolithiques renfermant des nanoparticules de ZnO. Journal de Physique IV Proceedings, 1996, 06 (C4), pp.C4-939-C4-945. 10.1051/jp4:1996491 . jpa-00254374

\section{HAL Id: jpa-00254374 https://hal.science/jpa-00254374}

Submitted on 1 Jan 1996

HAL is a multi-disciplinary open access archive for the deposit and dissemination of scientific research documents, whether they are published or not. The documents may come from teaching and research institutions in France or abroad, or from public or private research centers.
L'archive ouverte pluridisciplinaire HAL, est destinée au dépôt et à la diffusion de documents scientifiques de niveau recherche, publiés ou non, émanant des établissements d'enseignement et de recherche français ou étrangers, des laboratoires publics ou privés. 


\title{
Etude par EXAFS et fluorescence $X$ de photocatalyseurs zéolithiques renfermant des nanoparticules de $\mathrm{ZnO}$
}

\author{
L. Khouchaf*,**, M.H. Tuilier*, J. Dürr**, M. Wark*** et H. Kessler*** \\ * Laboratoire de Physique et de Spectroscopie Electronique, URA 1435 du CNRS, UHA, 4 rue des Frères \\ Lumière, 68093 Mulhouse cedex, France \\ ** Laboratoire d'Analyse Physique, Département Matériaux, Ecole des Mines de Douai, 59508 Douai \\ cedex, France \\ *** Laboratoire de Matériaux Minéraux, URA 428 du CNRS, ENSCM, 3 rue Alfred Werner, \\ 68093 Mulhouse cedex, France
}

\begin{abstract}
Résumé: Nous présentons les premiers résultats obtenus par spectroscopie d'absorption $\mathrm{X}$ au seuil $\mathrm{K}$ du zinc et d'émission X sur la structure atomique de clusters formés à partir de zinc et d'oxygène, confinés à l'intérieur des cavités de la sodalite.

Nous avons commencé par une étude de la structure des références $\mathrm{ZnAl}_{2} \mathrm{O}_{4}$ et $\mathrm{ZnO}$. Les résultats obtenus sont en bon accord avec les données cristallographiques. Dans le cas des échantillons de sodalite, le zinc est entouré en premiers voisins par de l'oxygène dans un environnement tétraédrique; la distance $\mathrm{Zn}-\mathrm{O}$ est de $1.97 \AA$. En seconds et troisièmes voisins le zinc est entouré par de l'aluminium et du zinc respectivement à des distances $\mathrm{Zn}-\mathrm{Al}$ de $2.86 \AA$ et $\mathrm{Zn}-\mathrm{Zn}$ de $3.38 \AA$. Une étude de l'influence de la température de calcination a également été faite. Ces résultats ont permis la mise en évidence d' une forte interaction des clusters avec la charpente.
\end{abstract}

\begin{abstract}
The first results on ZnK X-ray emission and Zn K EXAFS studies of ZnO clusters confined inside the cages of sodalite are reported here.

The analysis of the first, second and third shells of coordination around $\mathrm{Zn}$ in $\mathrm{ZnO}$ and $\mathrm{ZnAl}_{2} \mathrm{O}_{4}$ references is presented. The results agree fairly well with the crystallographic data. In sodalite samples, the $\mathrm{Zn}$ atoms are in tetrahedral coordination with oxygen at $1.97 \AA$. The second and third neighbours of $\mathrm{Zn}$ are $\mathrm{Al}$ at $2.86 \AA$ and $\mathrm{Zn}$ at $3.38 \AA$ respectively, the former belonging to the sodalite framework. The influence of the calcination temperature on the local order around $\mathrm{Zn}$ is also discussed. Interaction between the clusters and the framework is clearly evidenced.
\end{abstract}

\section{INTRODUCTION}

Les propriétés physiques (en particulier optoélectroniques) de certains composés, peuvent être modifiées suite à leur inclusion à l'intérieur des cavités de faibles dimensions telles qu'il en existe dans certains variétés de zéolithes dont la sodalite $[1,2]$. La maille élémentaire de la sodalite cubique est formée par l'assemblage de deux cages sodalite de diamètre interne $6.6 \AA$ chacune [1]. Une autre variété de zéolithe, la zéolithe A, est formée par des cages sodalite liées entre elles par l'intermédiaire de tétraèdres. Chaque combinaison de huit cages donne lieu à la formation d'une nouvelle cavité de plus grand diamètre interne de $11.3 \AA[3,4]$. La technique de l'EXAFS a permis d'étudier la structure de particules de très faibles dimensions incluses dans d'autres matériaux microporeux tels que la zéolithe- $Y$ et la Na-mordénite $[5,6]$.

Le traitement de sodalites et de zéolithes-A par une solution de $\mathrm{ZnCl}_{2}$ conduit à l'insertion d'ions complexes de $\mathrm{Zn}$ ou de clusters de structures variées dans les différentes cages et cavités de ces matériaux. La sodalite, qui possède uniquement des cages de faible dimension toutes identiques, constitue à cet égard un nouveau "modèle" pour l'étude de la localisation 
de très petites particules et de leur liaison avec la paroi. L'absorption $X$ et la fluorescence $X$ ont été utilisées pour la caractérisation de l'environnement du zinc inclus dans les cages de la sodalite. En particulier, l'analyse EXAFS a permis par l'étude des couches de coordination en premiers voisins (PV), seconds voisins (SV) et troisièmes voisins (TV) de préciser la structure d'un cluster formé par insertion du zinc dans les cages de la sodalite ainsi que leur mode d'interaction avec la charpente.

\section{EXPERIENCES}

La préparation de la sodalite de départ de formule $\mathrm{Na}_{6} \mathrm{Si}_{6} \mathrm{Al}_{6} \mathrm{O}_{24}, 8 \mathrm{H}_{2} \mathrm{O}$ sera décrite dans la référence [7]. Après traitement par une solution de $\mathrm{ZnCl}_{2}$, on prélève trois fractions de produit, de formule $\mathrm{Na}_{4.2} \mathrm{Zn}_{0.9} \mathrm{Si}_{6} \mathrm{Al}_{6} \mathrm{O}_{24}, \mathrm{xH}_{2} \mathrm{O}$, qui sont séchés à $80^{\circ} \mathrm{C}, \mathrm{x}=12$ (SOD80), et calcinés à $400^{\circ} \mathrm{C}, \mathrm{x}=2$ (SOD400), et à $600^{\circ} \mathrm{C}, \mathrm{x}=2$ (SOD600) respectivement pendant plusieurs heures.

Les spectres de fluorescence $X$ ont été obtenus à température ambiante, sous vide primaire $\left(10^{-2} \mathrm{mbar}\right)$ en utilisant un spectromètre séquentiel de fluorescence $\mathrm{X}$, de type Siemens $(60$ $\mathrm{kV}, 80 \mathrm{~mA}$ ) [8] ; Le pas entre deux mesures successives est de $0.016^{\circ}$ en $2 \theta$. Le test de résolution et de stabilité a été effectué à l'énergie de la raie d'émission du fer FeK $\beta$ dans des oxydes de fer tels que $\mathrm{FeO}, \mathrm{Fe}_{3} \mathrm{O}_{4}, \mathrm{Fe}_{2} \mathrm{O}_{3}$ et le fer métal, en utilisant les cristaux analyseurs $\mathrm{LiF}(100)$ et $\mathrm{Si}(511)$. Pour les raies d'émission $\mathrm{ZnK} \beta$ le LiF(110) a été utilisé [8].

Les expériences EXAFS ont été réalisées au Laboratoire pour 1'Utilisation du Rayonnement Electromagnétique (Orsay, France) sur la ligne de faisceau XAS2 de l'anneau de stockage DCI avec une énergie de positrons de $1.85 \mathrm{GeV}$ et un courant de $320 \mathrm{~mA}$. L'enregistrement des données à $77 \mathrm{~K}$ a été effectué en mode de transmission au seuil $\mathrm{K}$ du zinc $\left(\mathrm{E}_{\text {seuil }}=9659\right.$ $\mathrm{eV}$ ) dans la région d'énergies $(9500-10500 \mathrm{eV})$ en utilisant un spectromètre à deux cristaux de silicium taillés selon le plan de réflexion (311) Les intensités incidente et transmise, ont été mesurées en utilisant deux chambres d'ionisation remplies par de l'argon. Les échantillons sous forme de poudres ont été mélangés avec une faible quantité de cellulose, puis comprimés entre deux feuillets de capton. L'épaisseur des échantillons a été ajustée afin d'obtenir un saut d'absorption $\Delta \mu$ de l'ordre de 1 .

Afin d'extraire des informations structurales des données EXAFS, le fond continu d'absorption avant et après seuil a été soustrait des spectres d'absorption $\mu(E)$ en fonction de l'énergie des photons $E$ en utilisant une fonction linéaire ou une fonction de Victoreen [9]. Après la conversion de $\chi(\mathrm{E})$ en $\chi(\mathrm{k})$, la partie oscillante (oscillations EXAFS) est obtenue en calculant la quantité exprimée en fonction de $\mathrm{k}$ :

$$
\chi(k)=\frac{\mu(k)-\mu_{0}(k)}{\mu_{0}(k)}
$$

où $\mu_{0}(\mathrm{k})$ et $\mu(\mathrm{k})$ représentent respectivement le coefficient d'absorption de l'atome isolé, et celui de l'atome en présence de voisins, et $\mathrm{k}$ le vecteur d'onde du photoélectron définie comme :

$$
k=\frac{\sqrt{2 m\left(E-E_{0}\right)}}{\hbar}
$$


où $E_{0}$ est l'énergie seuil. Le choix de $E_{0}$ sur le spectre expérimental, consiste à la détermination du point qui correspond à une énergie cinétique nulle du photoélectron. On a choisi le même point (bas de la marche d'absorption) sur les spectres d'absorption pour les références et les échantillons. Les spectres EXAFS résultants sont amplifiés d'un facteur $\mathrm{k}^{2}$ dans la région 2.5-12 $\AA^{-1}$ avant de calculer leurs transformées de Fourier (TF). La simulation des transformées de Fourier inverses (TFI) des PV, SV et TV est effectuée dans le cadre du formalisme de diffusion simple en utilisant la formule du coefficient de structure fine :

$$
\chi(k)=-\sum_{i} \frac{N_{i}}{k R_{i}^{2}} F_{i}(k, \pi) \exp \left(-2 \sigma_{i}^{2} k^{2}\right) \exp \left(-\frac{2 R_{i}}{\lambda(k)}\right) \sin \left(2 k R_{i}+\phi_{i}(k)\right)
$$

avec $\mathrm{N}_{\mathrm{i}}$ le nombre de voisins autour de l'atome émetteur, $\mathrm{R}_{\mathrm{i}}$ la distance qui sépare l'atome émetteur et l'atome diffuseur, $\mathrm{F}_{\mathrm{i}}(\mathrm{k}, \pi)$ et $\phi_{i}(k)$ désignent respectivement l'amplitude de rétrodiffusion et le déphasage total, $\sigma_{i}$ le facteur de Debye-Waller lié aux vibrations thermiques et au désordre structural, $\lambda(k)$ le libre parcours moyen du photoélectron dans la matière. Les déphasages théoriques [10] ont été utilisés pour la simulation des références $\mathrm{ZnAl}_{2} \mathrm{O}_{4}$ et $\mathrm{ZnO}$.

\section{RESULTATS ET DISCUSSIONS}

\subsection{Fluorescence $X$}

Les raies d'émission $\mathrm{ZnK} \beta_{13}$ des échantillons de sodalite sont déplacées vers les faibles énergies par rapport à celles de $\mathrm{ZnO}$ et de $\mathrm{ZnAl}_{2} \mathrm{O}_{4}$. Les déplacement sont respectivement de $-0.1 \pm 0.1 \mathrm{eV}$ pour SOD400 et de $-0.3 \pm 0.1 \mathrm{eV}$ pour $\mathrm{SOD} 600$ par rapport à $\mathrm{ZnAl}{ }_{2} \mathrm{O}_{4}$ et $\mathrm{ZnO}$. Ces déplacements, bien qu'ils soient faibles, peuvent être attribués à un environnement local différent dans les sodalites traitées et dans les oxydes de référence $\mathrm{ZnAl}_{2} \mathrm{O}_{4}$ et $\mathrm{ZnO}$. L'étude EXAFS permet donc d'apporter des informations structurales plus précises.

\subsection{EXAFS}

\subsubsection{Références: $\mathrm{ZnAl}_{2} \mathrm{O}_{4}$ et $\mathrm{ZnO}$}

Sur la figure 1 sont présentés les spectres EXAFS des deux références : l'aluminate de zinc $\mathrm{ZnAl}_{2} \mathrm{O}_{4}$ de structure spinelle et l'oxyde de zinc $\mathrm{ZnO}$ qui cristallise dans le système hexagonal. Dans ces deux références, le zinc est dans un environnement tétraédrique d'oxygène à une distance moyenne de $1.96 \AA[11,12]$, ce tétraèdre est légèrement distordu dans $\mathrm{ZnO}$. Dans $\mathrm{ZnAl}_{2} \mathrm{O}_{4}$, le zinc est entouré par $12 \mathrm{Al}$ comme seconds voisins à une distance moyenne de $3.35 \AA$, et de $4 \mathrm{Zn}$ comme troisièmes voisins à une distance moyenne de $3.50 \AA$. Dans l'oxyde de zinc, le zinc est entouré par $12 \mathrm{Zn}$ comme seconds voisins à une distance moyenne de $3.23 \AA$, et de 9 atomes d'oxygène comme troisièmes voisins à une distance moyenne de $3.81 \AA$ A. Sur la figure 2 sont représentés les TF des spectres EXAFS. Nous avons calculé la TFI de chacun des différents pics et avons simulé les données par la formule de l'EXAFS. Les résultats obtenus sont résumés dans le tableau 1. Nous constatons un bon 
accord entre les données cristallographiques et les résultats EXAFS. Ces deux références seront utilisées pour extraire les phases et amplitudes de rétrodiffusion afin de simuler les PV oxygène et les voisins zinc dans les échantillons inconnus.

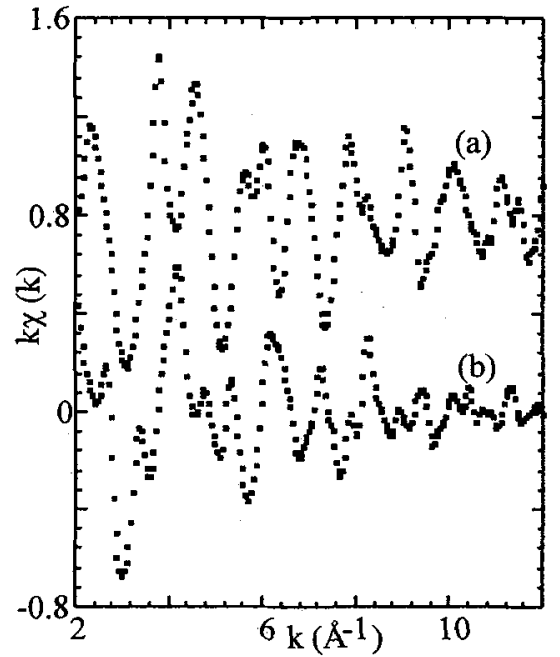

Figure.1: Spectres EXAFS $\mathrm{k} \chi(\mathrm{k})$ au seuil $\mathrm{K}$ du zinc dans (a) $\mathrm{ZnO}$; (b) $\mathrm{ZnAl}_{2} \mathrm{O}_{4}$.

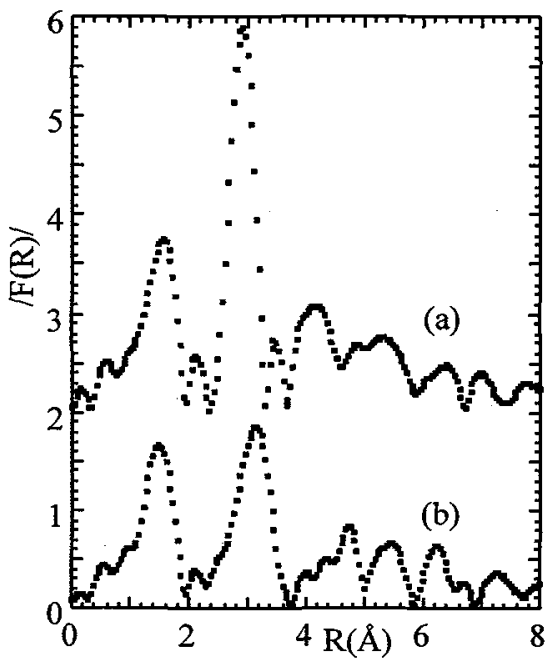

Figure.2: Transformées de Fourier des spectres EXAFS dans (a) $\mathrm{ZnO}$; (b) $\mathrm{ZnAl}_{2} \mathrm{O}_{4}$.

Tableau.1: Comparaison des données cristallographiques avec celles obtenues par EXAFS dans les références.

\begin{tabular}{|c|c|c|c|c|c|c|c|c|c|}
\hline Composé & TR1 & $\mathrm{N}_{1}$ & $\mathrm{R}_{1}(\AA)$ & $\mathrm{TR} 2$ & $\mathrm{~N}_{2}$ & $\mathrm{R}_{2}(\AA)$ & TR3 & $\mathrm{N}_{3}$ & $\mathrm{R}_{3}(\AA)$ \\
\hline EXAFS & $\mathrm{O}$ & 4 & $1.97 \pm 0.01$ & - & - & - & $\mathrm{Zn}$ & 12 & $3.24 \pm 0.02$ \\
\hline Cristallographie $^{\mathrm{a}}$ & $\mathrm{O}$ & 4 & 1.97 & $\mathrm{O}$ & 1 & 3.22 & $\mathrm{Zn}$ & 12 & 3.23 \\
\hline \multicolumn{10}{|l|}{$\mathrm{ZnAl}_{2} \mathrm{O}_{4}{ }^{\text {EXAFS }}$} \\
\hline Cristallographie ${ }^{b}$ & $\mathrm{o}$ & 4 & 1.95 & $\mathrm{Al}$ & 12 & 3.35 & $\mathrm{Zn}$ & 4 & 3.50 \\
\hline
\end{tabular}

TR: Type de rétrodiffuseur; a) Ref 11, b) Ref 12 
3.1.2 Echantillons de sodalites calcinés à $80^{\circ} \mathrm{C}(S O D 80), 400{ }^{\circ} \mathrm{C}(S O D 400)$, et $600{ }^{\circ} \mathrm{C}$ (SOD600)

Les spectres EXAFS des échantillons SOD80, SOD400 et SOD600 sont représentés sur la figure 3. La séparation des fréquences de ces oscillations et donc la contribution des différents voisins autour du zinc est représentée sur la figure 4. Chacun des spectres présente plusieurs pics que nous allons décrire successivement.

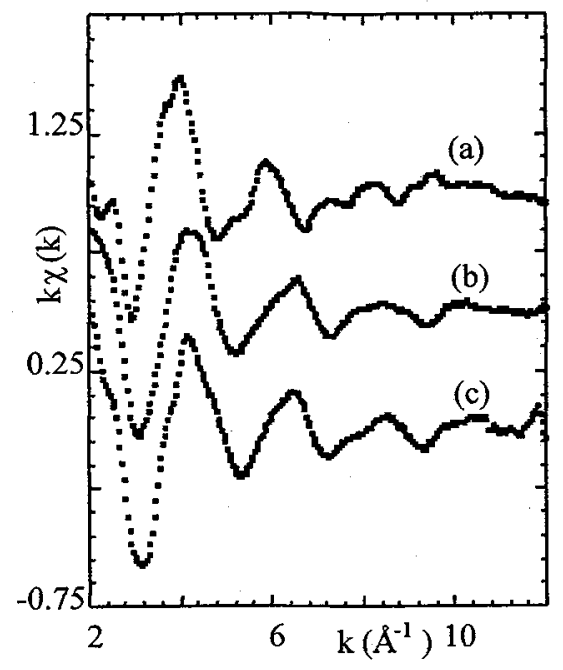

Figure.3: Spectres EXAFS $\mathrm{k} \chi(\mathrm{k})$ au seuil $\mathrm{K}$ du zinc dans les échantillons de sodalite (a) SOD80; (b) SOD400; (c) SOD600.

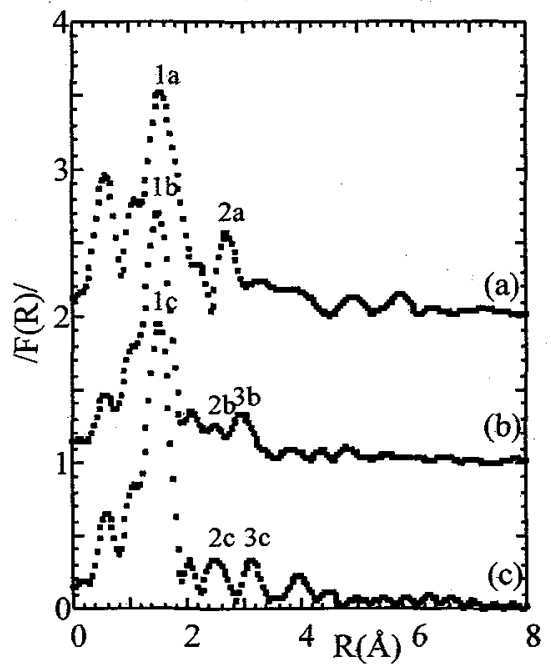

Figure.4: Transformées de Fourier des spectres EXAFS dans les échantillons de sodalite (a) SOD80; (b) SOD400; (c) SOD600.

3.1.2.1 Premiers voisins La modélisation de la TFI du premier pic dans les trois échantillons de sodalite en utilisant les phases et amplitudes expérimentales de $\mathrm{ZnO}$ et de $\mathrm{ZnAl}_{2} \mathrm{O}_{4}$ a montré qu'il s'agit de premiers voisins oxygène dans un environnement tétraédrique autour du zinc à une distance moyenne de $1.96 \pm 0.01 \AA$ dans SOD400 et SOD600 et de $2.09 \pm 0.01 \AA$ dans SOD80. Il est à noter que dans le cas de SOD80, ce pic de premiers voisins est plus étendu comparé à ceux de SOD400 et SOD600. Cet effet se traduit par un facteur de DebyeWaller plus important dans cet échantillon. Elle a pour origine une distorsion importante du polyèdre de coordination du zinc par rapport aux références.

3.1.2.2 Seconds et troisièmes voisins Au delà des premiers voisins, la comparaison entre les sodalites calcinées à différentes températures montre une différence nette dans les spectres de $\mathrm{TF}$.

Le spectre TF de la SOD80 (fig. 4a) présente un seul pic (pic2a) au delà du pic de PV. L'analyse et la modélisation de ce deuxième pic nous ont permis de montrer qu'il s'agit de 
seconds voisins zinc situés à une distance moyenne de $3.10 \AA$. Le détail du traitement sera présenté ultérieurement [7].

Dans le cas de la SOD400, deux pics apparaissent au-delà du pic des PV (fig. 4b) : l'un de faible amplitude autour de $2.5 \AA$ (pic2b) et l'autre à $3.3 \AA$ (pic3b). Malgré sa faible amplitude, nous verrons dans la suite que le pic $2 b$ est très significatif et nous avons pu l'identifier. La modélisation de la TFI du pic3b successivement par la phase et l'amplitude de rétrodiffusion du zinc dans $\mathrm{ZnO}$ et théoriques [10] nous a permis de l'attribuer à des voisins zinc situés à une distance moyenne de $3.32 \AA[$ [7].

Le spectre TF de l'échantillon SOD600 présente trois pics au delà du pic de PV (fig. 4c). Cela montre qu'il y a un ordre local plus important autour du zinc dans cette échantillon. Un fait très important à remarquer est l'augmentation de l'amplitude de rétrodiffusion du pic2c. Cela facilite alors son exploitation. L'analyse de la forme de son amplitude de rétrodiffusion montre bien qu'il s'agit d'un voisin léger. Or, les éléments légers que contient l'échantillon sont : $\mathrm{O}, \mathrm{Na}, \mathrm{Al}$ et $\mathrm{Si}$. La modélisation avec la phase et l'amplitude de rétrodiffusion de chacun de ces éléments nous a conduit finalement à l'attribuer à des voisins aluminium ou silicium de la charpente à une distance moyenne de $2.86 \AA$, plus faible que celle dans $\mathrm{ZnAl}_{2} \mathrm{O}_{4}$. Ce résultat mis en évidence une interaction du zinc avec la charpente.

De même, nous avons identifié le pic $3 \mathrm{c}$ à des voisins zinc localisés à une distance moyenne de $3.38 \AA$, plus longue que celle dans $\mathrm{ZnO}$. Ces résultats sont en faveur de l'inclusion de clusters de $\left(Z n_{4} O\right)^{6+}$ à l'intérieur des cages sodalites [7]. Dans ce modèle d'inclusion de clusters $\left(\mathrm{Zn}_{4} \mathrm{O}\right)^{6+}$ à l'intérieur des cages sodalites, l'environnement tétraédrique du zinc est formé d'une liaison avec un atome d'oxygène situé au centre de la cage sodalite, et trois autres avec trois oxygènes de la charpente; ce cluster est donc en interaction avec la charpente. Cette interaction est de plus en plus marquée quand la température de calcination est élevée. Elle est différente dans la SOD80, ce qui est en bon accord avec la faible distance $\mathrm{Zn}-\mathrm{Zn}$ obtenue dans cette échantillon. Cette différence est due à une absence des contributions de voisins $\mathrm{Al}$ ( $\mathrm{Si}$ ), sans doute puisque les distances $\mathrm{Zn}-\mathrm{Al}(\mathrm{Zn}-\mathrm{Si})$ sont, elles, trop dispersées et leur contribution au spectre EXAFS est faible.

\section{CONCLUSION}

A travers cette étude nous avons pu déterminer l'environnement local autour du zinc confiné à l'intérieur des cages sodalites. Le zinc est dans un environnement tétraédrique d'oxygène à une distance moyenne de $1.96 \AA$. En seconds et troisièmes voisins il est entouré par de l'aluminium et du zinc. Lorsque la température de calcination s'élève à $400^{\circ} \mathrm{C}$, nous avons montré une forte interaction du zinc avec la charpente. Ces résultats sont en bon accord avec le modèle d'inclusion de nanoparticules de $\left(\mathrm{Zn}_{4} O\right)^{6+}$ à l'intérieur des cages sodalites. Ils seront comparés dans une autre étude à ceux d'absorption UV-visible afin de confirmer la structure de cet ion complexe. Par ailleurs, ce travail est une étude préliminaire de l'environnement local du zinc dans des matériaux microporeux tels que des zéolithes A de structure plus complexe qui présentent deux types de pores : des cages sodalite et des cavités de plus grande dimension. 


\section{Références}

[1] Meier W. M. et Olson D. H., Atlas of zeolite structure types ( Butherworth-Heinemann, 1992) pp. 172-173.

[2] Moran K. L. et al., J. Am. Chem. Soc. 115, (1993) 10553.

[3] Meier W. M. et Olson D. H., Atlas of zeolite structure types ( Butherworth-Heinemann, 1992) pp. 122-123.

[4] Break D. W., Zeolite Molecular Sieves ( John Wiley, New york, 1974) pp. 83-85.

[5] Moller K. et al, J. Am. Chem. Soc. 111 (1989) 2564.

[6] L. Khouchaf et al., J. Phys. Chem. Solids, sous presse (1996).

[7] L. Khouchaf et al. (à paraître).

[8] Khouchaf L.et Dürr J., Rapport interne de l'Ecole Nationale Supérieure des Mines de Douai, (1995).

[9] Michalovicz A., Logiciels pour la chimie (Société Française de Chimie, Paris, 1991) pp. 102-103.

[10] A. G. Mc Kale et al., J. Am. Chem. Soc. 110 (1988) 3763.

[11] Abrahams S. C. et Bernstein J. L., Acta. Cryst.25.(1969) 1233.

[12] Fischer P., Zeitschr. Kris. 124 (1967) 275-302. 